\title{
Impacto da pandemia do SARS-CoV-2 na ocupação e mobilização de pacientes de uma unidade de terapia intensiva cardiovascular
}

\author{
The impact of SARS-CoV-2 pandemic on the occupation and mobilization of patients in \\ a cardiovascular intensive care unit
}

José da Natividade Menezes Júnior ${ }^{1 *}$, Helena França Correia²

${ }^{1}$ Fisioterapeuta. Mestrando do Programa de Pós Graduação Processos Interativos do Orgãos e Sistemas. Instituto de Ciencias da Saúde, Universidade Federal da Bahia - UFBA. Supervisor de Fisioterapia da UTI Cardiovascular do Hospital Geral Roberto Santos; ${ }^{2}$ Doutora em Medicina e Saúde Humana. Professora associada. Programa de Pósgraduação em Processos Interativos do Orgãos e Sistemas. Instituto de Ciências da Saúde - UFBA

\begin{abstract}
Resumo
Introdução: a pandemia do SARS-CoV-2 causou impactos econômicos e sociais em todo o mundo, em especial nos sistemas de saúde, repercutindo no número de admissões hospitalares por outras doenças, na rotina dos cuidados, no acesso aos serviços e nos recursos materiais, humanos e financeiros disponibilizados. Objetivo: Analisar o impacto da pandemia SARS-CoV-2 no número de internamentos e mobilização de pacientes críticos de uma unidade de terapia intensiva cardiovascular. Metodologia: trata-se de um estudo transversal, retrospectivo, realizado na Unidade de Terapia Intensiva Cardiovascular do Hospital Geral Roberto Santos, Salvador-BA. Analisaram-se comparativamente os dados de internamento e mobilização dos pacientes, no período entre março de 2019 e fevereiro de 2020, isto é pré-pandemia, e o primeiro ano de sua ocorrência. Resultados: houve redução $(p<0,001)$ no número de internações no setor, quanto a mobilização, embora impactada nos primeiros meses da pandemia, conseguiu manter dados estáveis, quando avaliada de maneira linear. Discussão: A redução na ocupação coincide com achados de estudos nacionais e internacionais, o que pode ter influência na orientação de distanciamento social e o receio de contágio pelo vírus. Quanto à mobilização, importante instrumento de atuação fisioterapêutica nas UTI, apresentou dados similares $(p=0,8)$, quando comparados os dois períodos do estudo. Conclusão: notou-se impacto da pandemia no número de internamentos em uma UTI cardiológica durante a pandemia, o que deve ser futuramente estudado para avaliar as reais consequências dessa redução assistencial. Quanto à mobilização, não foi observada alteração significativa na média, antes e após a pandemia.
\end{abstract}

Palavras-chave: COVID 19. SARS-CoV-2. Mobilização Precoce. Hospitalização. Cardiologia.

\begin{abstract}
Introduction: SARS-CoV-2 pandemic caused economic and social impacts around the world, especially in health systems, affecting the number of hospital admissions for other diseases, routine care, access to services and material and human resources and financial resources made available. Objective: to analyze the impact of SARS-COV-2 pandemic on the number of admissions and mobilization of critically ill patients in a cardiovascular intensive care unit. Methodology: this is a cross-sectional, retrospective study carried out at the Cardiovascular Intensive Care Unit of Hospital Geral Roberto Santos, Salvador-BA. Data on hospitalization and patient mobilization between March 2019 and February 2020 (pre-pandemic) and during the first year of its occurrence were comparatively analyzed. Results: there was a reduction $(p<0.001)$ in the number of hospitalizations in the sector and mobilization, although impacted in the first months of the pandemic, managed to maintain stable data when evaluated in a linear fashion. Discussion: the reduction in occupation coincides with findings from national and international studies, which may influence the orientation of social distancing and the fear of contagion by the virus. As for mobilization, an important instrument of physical therapy practice in the ICUs, it presented similar data ( $(p=0.8)$, when comparing the two periods of the study. Conclusion: the impact of the pandemic was noted on the number of admissions to a cardiology ICU during the pandemic, which should be studied in the future to assess the real consequences of this reduction in care. As for mobilization, there was no significant change in the mean, before and after the pandemic.
\end{abstract}

Keywords: COVID 19. SARS-CoV-2. Early Mobilization. Hospitalization. Cardiology

\section{INTRODUÇÃO}

No final de 2019, o mundo tomou conhecimento do surgimento do SARS-CoV-2, do qual resultou uma crise de saúde não testemunhada desde a pandemia

Corrrespondente/Corresponding: *José da Natividade Menezes Júnior - Setor de Fisioterapia do Hospital Geral Roberto Santos - End: Rua Direta do Saboeiro, s/n - Cabula, 40301-110 - Salvador - BA - Tel: 98871-5661 - E-mail: jose.natividade@ufba.br de influenza na Espanha de 1918-1919. A origem mais plausível do SARS-CoV-2 é a seleção natural do vírus em um hospedeiro animal, seguida por transferência zoonótica. Após os primeiros casos de COVID-19 serem identificados em Wuhan, China, o vírus se espalhou rapidamente pelo mundo, sendo notificado em 220 países até 25 de abril de 2021 (ANDERSEN et al., 2020; OSUCHOWSKI et al., 2021). 
O Brasil está entre os países mais afetados pelo COVID-19, em paralelo aos Estados Unidos da América e à Índia, totalizando mais de 1,6 milhão de mortes e 88 milhões de infectados até agosto de 2021 (COVID-19 coronavirus pandemic - Worldmeter, 2021), apesar da taxa de mortalidade marcadamente mais baixa do COVID-19, em comparação com as outras epidemias de coronavírus, como a síndrome respiratória aguda grave (SARS) e a síndrome respiratória do Oriente Médio (MERS) (OSUCHOWSKI et al., 2021). A resposta das comunidades médica e científica foi sem precedentes, sendo que foram produzidos um número extraordinário de relatórios de pesquisa relacionados com SARS-CoV-2 e COVID-19 (ALWAN et al., 2020).

Ao analisar de maneira mais minuciosa os desdobramentos gerados pela infecção do SARS-CoV-2, observou-se que os seus efeitos no sistema de saúde impactaram diretamente no acesso para pacientes portadores de outras patologias, visto que grande parte dos recursos materiais, humanos e financeiros foram direcionados para o enfrentamento da pandemia (LANA et al., 2020). Estudos realizados em outras populações mundiais apontam para a redução no número de admissões hospitalares por doenças cardiovasculares (DCV) (TONIOLO et al., 2020). Em estudo que avaliava o impacto no acesso à saúde por parte da população brasileira, foi identificada limitação de acesso a serviços de saúde, tais como marcação de consultas e exames e procedimentos cirúrgicos com o surgimento da pandemia (SZWARCWALD et al., 2020). Nesse cenário, destacam-se as DCV, por seu caráter também pandêmico, visto que esse grupo de afecções é uma das principais causas de mortalidade em todo mundo, principalmente em países de baixa e média renda, como é o caso do Brasil. Na Bahia e demais estados da região Nordeste, o impacto dessas patologias é ainda maior, pois as taxas de mortalidade se mantêm em elevação em comparação a outros estados brasileiros, a exemplo das regiões Sul e Sudeste (MANSUR; FAVARATO, 2016; ROTH et al., 2018).

A mobilização é parte do processo de reabilitação dos pacientes internados em unidade de terapia intensiva (UTI), recentemente considerada como uma forma de prevenção da fraqueza muscular adquirida pelo imobilismo e da piora da função física. Trata-se de uma terapêutica de responsabilidade do fisioterapeuta, o qual define o melhor modelo de intervenção, sua intensidade, periodicidade, continuidade ou interrupção, com o objetivo de trazer benefícios fisiológicos e de auxiliar na recuperação funcional. Compreende exercícios físicos voltados para a sedestração a beira do leito, transferência para a cadeira, ortostatismo e deambulação (ADLER; MALONE, 2012; BURTIN et al., 2009).

As consequências do imobilismo estão associadas à idade avançada, à gravidade da doença, ao diagnóstico clínico e à severidade da doença. Os seus efeitos podem se estender até cinco anos após a alta hospitalar, contribuindo para o declínio da capacidade funcional desses indivíduos, sendo, portanto, a mobilização precoce um importante instrumento terapêutico na prevenção de danos e na manutenção do desempenho funcional (NEEDHAM et al., 2010).

Nos pacientes cardiopatas, a mobilização precoce é extremamente importante no processo de reabilitação. Atualmente, é corriqueiro nas UTI a realização de testes para avaliação do desempenho funcional, tais como: teste de caminhada de seis minutos (TC6M), teste de velocidade da marcha (TVM) e o teste de sentar e levan$\operatorname{tar}(T S L)$, pois são relevantes mecanismos de avaliação usados para formular estratégias de prevenção, reabilitação, determinar prognóstico e detectar indivíduos com maior vulnerabilidade ao declínio funcional. Trata-se de instrumentos práticos que traduzem não apenas a capacidade funcional e o estado geral de saúde, mas também têm sido utilizados em estudos que avaliam equilíbrio e mobilidade, antes, durante e após programas de reabilitação de pacientes portadores de cardiopatias (AMERICAN TORACIC SOCIETY, 2002; GARCIA et al., 2012; GRUET et al,. 2016).

Diante disso, o presente estudo teve por objetivo analisar o impacto da pandemia SARS-CoV-2 no número de internamentos e na mobilização precoce de pacientes críticos em uma unidade de terapia intensiva cardiovascular.

\section{METODOLOGIA}

Trata-se de um estudo transversal, retrospectivo, realizado na Unidade de Terapia Intensiva Cardiovascular de um hospital público na cidade de Salvador-BA. Analisaram-se o número de internações e o perfil de mobilização dos pacientes que receberam atendimento do serviço de Fisioterapia durante o período de internação na UTI cardiovascular, sendo excluídos aqueles com amputação em membros inferiores, lesões osteoarticulares, bem como os acometidos de sequelas neurológicas prévias que inviabilizaram a mobilização.

Os dados do número de internamentos no setor a cada mês, do percentual de mobilização e deambulação dos pacientes foram extraídos das planilhas dos indicadores assistenciais do setor de qualidade do serviço de fisioterapia do hospital, sendo considerados como mobilização: sedestração no leito com membros inferiores (MMII) pendentes, sedestração em poltrona e treino funcional da marcha, durante o internamento na UTI.

Com intuito de analisar de maneira comparativa o número de internamentos na unidade, bem como os indicadores de mobilização precoce e deambulação dos pacientes, estabeleceram-se como referência para o período pré-pandemia os 12 meses anteriores à declaração da OMS sobre sua expansão, que se deu em 11 de março de 2020; portanto, março de 2019 a fevereiro de 2020 foi o período considerado para a comparação com os 12 primeiros meses da pandemia do SARS-CoV-2, isto é, março de 2020 a fevereiro de 2021. 
As variáveis categóricas foram expressas em frequências absolutas e relativas. As variáveis numéricas foram expressas em média e desvio padrão, $\mathrm{O}$ teste $\mathrm{t}$ de Student foi utilizado para estabelecer a significância estatística da diferença entre as médias das admissões e o percentual de mobilização nos períodos pré-pandemia e pandemia. O nível de significância adotado foi de $\mathrm{p}<$ 0,05 . O tratamento estatístico foi realizado utilizando-se o Statistical Package for the Social Sciences, versão 21.0 (SPSS Inc., Chicago, IL, EUA).

O estudo foi aprovado pelo Comitê de Ética e Pesquisa do Hospital Geral Roberto Santos (CEP-HGRS), sob o Parecer n. 4.793.403, sendo que houve dispensa do Termo de Consentimento Livre e Esclarecido (TCLE), em virtude de o presente estudo ter usado como fonte da pesquisa apenas dados consolidados do setor de qualidade do serviço de Fisioterapia do hospital, sem acesso aos dados pessoais ou aos prontuários dos pacientes.

\section{RESULTADOS}

A média do número de admissões por mês, nos primeiros 12 meses da pandemia, foi significativamente menor que nos 12 meses anteriores à pandemia $(108,1 \pm 16,2$ vs $134,2 \pm 10,7$ pacientes/mês; $p<0,001)$. Os dados da evolução do número de internações pode ser observado na Figura 1.

Figura 1 - Evolução do número de admissões de março de 2019 a fevereiro de 2021

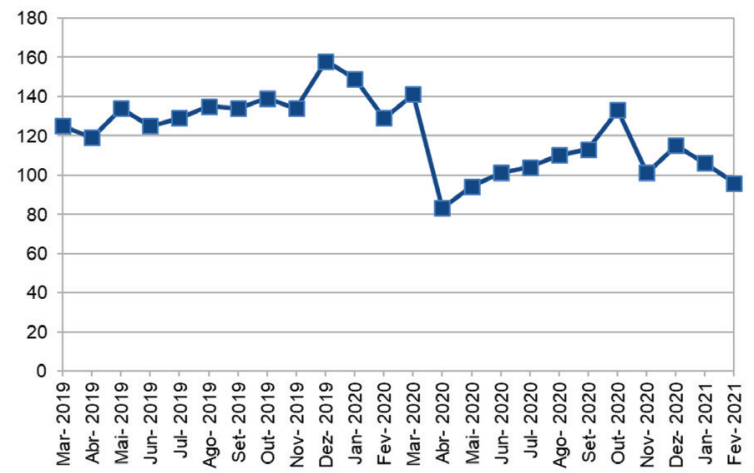

Fonte: Dados da pesquisa.

Não foram observadas diferenças ao se comparar as médias do percentual de pacientes mobilizados por mês entre os períodos pré-pandemia e os 12 primeiros meses da pandemia $(65,8 \pm 3,9 \%$ e $64,83 \pm 12,9 \%$; $p=0,80$; respectivamente). Na Figura 2 pode ser observado que houve uma tendência de queda nos três meses seguintes à declaração da pandemia pela OMS, mas logo essa tendência foi revertida, acarretando estabilidade dos dados quando comparados os dois períodos.
Figura 2 - Evolução do percentual de pacientes mobilizados (pacientes que realizaram sedestração no leito com MMII pendentes ou sedestração em poltrona, ou treino de ortostase ou deambularam), de março de 2019 a fevereiro de 2021.

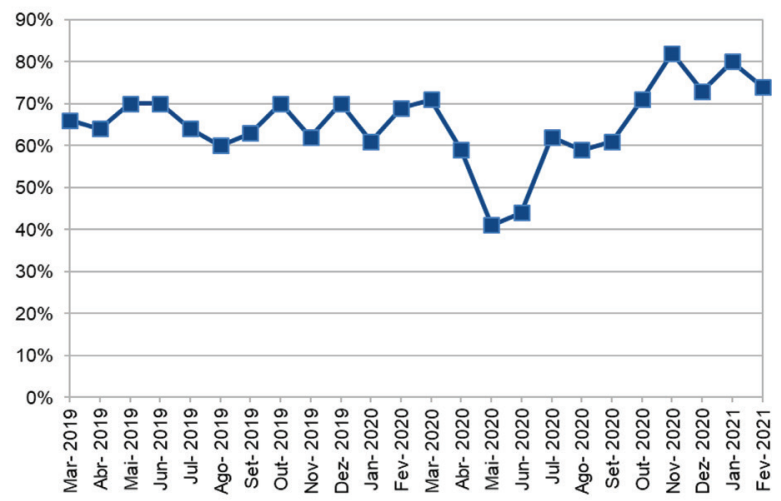

Fonte: Dados da pesquisa.

Em relação à média da taxa de pacientes que deambularam por mês, não foram encontradas diferenças significantes entre os dois períodos: $50,8 \pm 4,2 \%$, no período pré-pandemia,e $51,8 \pm 14,2 \%$, nos primeiros 12 meses da pandemia, $p=0,82$. Na Figura 3, observa-se um comportamento semelhante ao da mobilização nos três primeiros meses da pandemia do SARS-COV-2.

Figura 3 - Evolução do percentual de pacientes que deambularam de março de 2019 a fevereiro de 2021

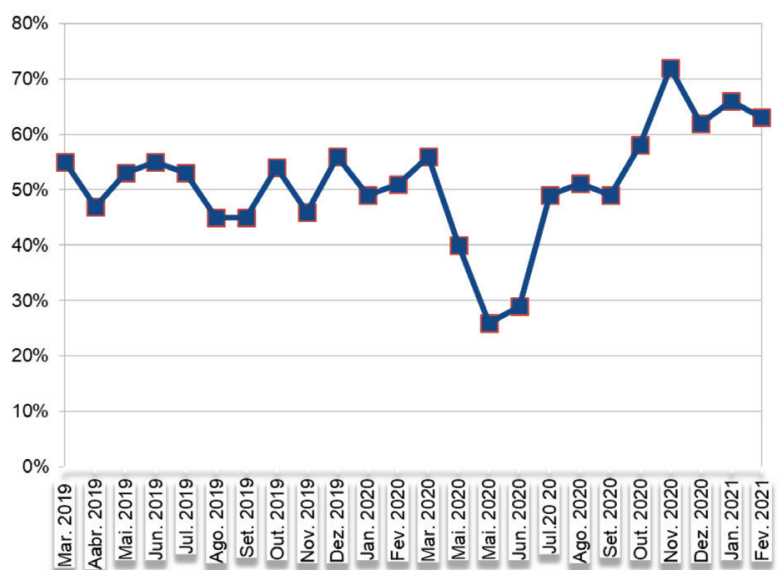

Fonte: Dados da pesquisa.

\section{DISCUSSÃO}

O presente estudo demonstra uma redução significativa no número de internações em uma UTI cardiovascular, com uma média $20 \%$ menor de admissões no período da pandemia, quando comparado ao ano anterior. No que tange à mobilização e à deambulação, observou-se uma redução pontual do percentual desses procedimentos nos primeiros três meses do evento, tendência logo revertida, possibilitando, assim, que o setor alcançasse médias equivalentes nos dois períodos estudados. 
No que tange à média de admissões do setor, o resultado obtido neste trabalho é um pouco superior ao encontrado em outro estudo (NORMANDO et al.,2021), no qual foi avaliada a redução das internações hospitalares por doenças cardiovasculares no Brasil, durante a pandemia do SARS-CoV-2, tendo como base os dados do Departamento de Informática do Sistema Único de Saúde (DATASUS), cujo impacto foi de $15 \%$ na busca por tratamento cardiológicos.

Estudo realizado na Itália (TONIOLO et al., 2020) observou uma redução importante $(51 \%)$ na busca por emergências cardiológicas na população de Veneza e outras regiões próximas, valores que corroboram os achados deste estudo no que diz respeito à queda de internaçoes; o referido estudo relacionou a queda na busca pelos servicos de saúde ao medo de contágio do vírus SARS-CoV-2, às notícias sobre pandemia nos meios de comunicação, bem como à disseminação de informações falsas em redes sociais, que levaram à percepção de que os hospitais e serviços de saúde eram inseguros.

A pandemia trouxe mudança no comportamento e nas atitudes em todo o mundo, sendo que o conhecimento das formas de transmissão gerou a instituição de estratégias de prevenção contra a disseminação do vírus e consequentemente a implementação de medidas de distanciamento social, que obrigaram milhões de pessoas a ficarem em seus domicílios, minimizando o contato com outras pessoas, impactando, desta forma, até mesmo na busca de cuidados com a saúde (CHU et al., 2020; ESTRELA et al., 2020; Ll et al., 2020).

Em pesquisa realizada no período entre abril a maio de 2020, ou seja, logo após o início da pandemia de COVID-19 no Brasil, notou-se essa abrupta queda na busca por assistência especializada, valores coincidentes aos encontrados no presente estudo. Embora pareça ser um curto período, determinadas doenças crônicas, principalmente aquelas descompensadas, dentre as quais estão as doenças cardiovasculares, podem apresentar uma piora exacerbada quando não manejadas adequadamente (MELO et al., 2020).

Os achados do presente trabalho corroboram o encontrado na literatura internacional, tais como em estudos realizados em países europeus e nos Estados Unidos da América, os quais revelam o impacto potencial no diagnóstico e no tratamento de condições clínicas em virtude da pandemia de COVID-19. Na Inglaterra, internações semanais por síndrome coronariana aguda apresentaram redução de cerca de $40 \%$ em março de 2020, depois apresentaram, assim como este estudo, uma reversão parcial, ficando com média de $16 \%$ em maio de 2020; nos EUA, os atendimentos em 24 prontos-socorros de cinco estados diminuíram entre 42 a $63 \%$, nos primeiros meses da pandemia (janeiro a abril de 2020) (JEFFERY et al., 2020; MAFHAM, 2020).

As medidas de distanciamento social levaram à modificação nos hábitos de vida, de uma maneira geral, e não foi diferente com os serviços de saúde, uma vez que os conselhos profissionais se apressaram em regulamentar o teleatendimento. A mudança de formato das consultas não resolveu o problema para a maioria dos usuários dos serviços de saúde no Brasil, já que não há acesso de uma parcela significativa da população em geral à internet de qualidade, que viabilize a realização das teleconsultas para acompanhamento de um profissional. Associado a este fato, parte da população com baixa renda tem dificuldade com o manejo tecnológico, sendo esta também uma importante barreira. Por ser uma ferramenta nova, há também questionamentos sobre o melhor modelo de abordagem para garantir a qualidade da anamnese e da avaliação física (BRINDA, 2020; GARCIA et al., 2020).

A redução do número de admissões na UTI cardiovascular, observada neste estudo, pode gerar um novo ciclo de alta referente às complicações cardíacas no período pós-pandemia, algo que deverá ser futuramente estudado para compreender as reais consequências na saúde pública. Normando et al. (2021) observaram que a assistência a patologias cardiovasculares tem sido adiada e/ou abreviada, sendo registrada uma maior taxa de letalidade hospitalar durante a pandemia, muito em decorrência do receio de contágio, bem como em virtude da mudança do perfil assistencial de muitos serviços de saúde, os quais se voltaram para assistência de pacientes com COVID-19, o que ocasionou redução do acesso ao serviço especializado. Não foi diferente com a cardiologia.

Deve-se destacar que as medidas de isolamento social trazem consequências psiquícas que acarretam transtornos de comportamento, sentimentos de tristeza pela perda de familiares, bem como o stress pela dificuldades para adaptação aos novos meios de interação social; associado à isso, a literatura evidencia alguns sintomas prevalentes, tais como: ansiedade, tédio, raiva, irritabilidade (FARO et al., 2020, WANG et al., 2020). Com base em estudos epidemiológicos, que proporcionam uma ampla literatura sobre o tema, pode-se afirmar que tais sintomas podem levar à precipitação de eventos cardíacos agudos (JIANG et al.,1996; LOURES et al,. 2002).

$\mathrm{Na}$ análise dos resultados, evidenciou-se estabilidade nos indicadores de mobilização no setor, com uma redução curta e pontual por dois meses (maio e junho 2020), mas logo revertida. Destaca-se, neste achado, a prevenção de danos no desempenho funcional, caso a mobilização, durante a internação hospitalar, tivesse sido reduzida, uma vez que esse procedimento faz parte do processo de cuidado, especificamente dos pacientes internados em unidades de terapia intensiva cardiovascular, por seu caráter de grande relevância para a prevenção de consequências do imobilismo, bem como preditor de possíveis complicações e reinternações nos testes funcionais (BUCKLEY et al., 2013; PRICE et al., 2016; RANDAL et al., 2019).

A atuação fisioterapêutica exerce um importante papel no fornecimento de intervenções para mobilização, principalmente em pacientes com risco de desenvolver declínio funcional (THOMAS et al., 2020). Cordeiro et al. 
(2015) analisaram o impacto da deambulação precoce em pacientes submetidos à cirurgia cardíaca, avaliaram os sinais vitais e encorajaram os indivíduos a caminharem uma distância de 100 metros, observando se ocorreria variação significativa de frequência cardíaca, frequência respiratória e do duplo produto ou eventos adversos associados; por fim, demonstraram que a deambulação é segura e viável dentro de unidades de terapia cardiovascular, se incorporada de maneira assertiva aos protocolos de reabilitação, tornando-se uma forma de prevenção da fraqueza muscular adquirida na UTI e da piora da função física e cardiorrespiratória.

Os achados do estudo mostraram que, além da redução no número de admissões, houve um impacto breve na mobilização precoce dos pacientes internados no setor, evidenciado a partir da detecção da redução de $30 \%$ dos pacientes que deambularam na unidade, quando comparados os meses de abril a junho de 2020 à media do setor pré-pandemia e no restante dos meses analisados. É claro na literatura especializada a importância da marcha porque ao tempo em que minimiza o risco de eventos adversos de segurança pode melhorar os resultados funcionais e se traduzir em redução do tempo de internação na UTI e no hospital (HODGSON et al., 2013).

O presente estudo, por ser observacional retrospectivo, apresenta como limitação principal possíveis falhas no preenchimento dos dados previamente consolidados; no entanto, como a equipe foi previamente treinada e contou diariamente com acompanhamento por parte do setor de qualidade e da supervisão do serviço de fisioterapia do hospital, visando a prevenção de falhas, os dados coletados apresentam uma precisão que permite ter-se confiança nos resultados.

\section{CONCLUSÃO}

Conclui-se que nos primeiros 12 meses da pandemia do SARS-COV-2 houve uma redução do números de internamentos em uma unidade de terapia instensiva cardiovascular, o que pode refletir uma redução na busca por acompanhamento cardiológico em um serviço público de referência. Quanto à mobilização e deambulação dos pacientes internados, notou-se que, após o impacto inicial, o setor apresenta dados semelhantes aos do período pré-pandemia.

\section{REFERÊNCIAS}

ADLER, J.; MALONE, D. Early mobilization in the intensive care unit: a systematic review. Cardiopulmonary Physical Therapy Journal, [s.I.], v. 23, n. 1, p. 23-25, 2012.

ALWAN, N. A. et al. Scientific consensus on the COVID-19 pandemic: we need to act now. Lancet, London, v. 396, n. 10260, p. E71-E72, 2020.

ANDERSEN, R. A et al. The proximal origin of SARS-CoV-2. Nature Medicine, New York, v. 26, n. 4, p. 450-452, 2020.

AMERICAN TORACIC SOCIETY. Committee on proficiency standards for clinical pulmonary function laboratories. ATS statement: Guidelines for the six minute walk test. Journal Respiratory Critical Care Medicine, New York, v. 166, n. 1, p. 111-117, 2002.

BINDA, D. L. B.; ZAGANELLI, M. V. Telemedicina em tempos de pandemia: serviços remotos de atenção à saúde no contexto da Covid-19. Humanidades e Tecnologia, [s.I.], v. 25, n. 1, p. 11-13, 2020.

BUCKLEY, J. P. et al. BACPR scientific statement: British standards and core components for cardiovascular disease prevention and rehabilitation. Heart, London, v. 99, n. 15, p. 1069-1071, 2013.

BURTIN, C. et al. Early exercise in critically ill patients enhances shortterm functional recovery. Critical Care Medicine, New York, v. 37, n. 9, p. 2499-2505, 2009.

CHU, D. K. et al. Physical distancing, face masks, and eye protection to prevent person-to-person transmission of SARS-CoV-2 and COVID - 19: a systematic review and meta-analysis. Lancet Public Health, [s.I.], v. 395, n. 10242, p. 1973-1987, 2020.

CORDEIRO, L. et al. Inspiratory muscle training and functional capacity in patients under going cardiac surgery. Brazilian Journal of Cardiovascular Surgery, São Paulo, v. 3, n. 2, p. 140, 2016.

COVID-19 coronavirus pandemic. Worldometer - real time world statistics. Disponível em: https://www.worldometers.info/coronavirus/. Acesso em: 5 ago. 2021.

ESTRELA, F. M. et al. Covid - 19 e Doenças crônicas: impactos e desdobramentos frente à pandemia. Revista Baiana de Enfermagen, Salvador, v. 34, n. 1,e36559, 2020.

FARO, A. et al. COVID-19 e saúde mental: a emergência do cuidado. Estudos de Psicologia, Natal, v. 37, n. 1, e200074, 2020.

GARCIA, M. V. F.; GARCIA, M. A. F. Telemedicine, legal certainty, and covid-19: Where are we? Jornal Brasileiro de Pneumologia, Brasília, v. 46, n. 4, p. 1-2, 2020.

GARCIA, G. et al. Avaliaçao da Indepedência funcional de pacientes pós-internados em unidade de terapaia intensiva. Conscientiae Saúde, São Paulo, v. 11, n. 2, p. 296-304, 2012.

GRUET, M. et al. The 1Minute sit-to-stand test in adults with cystic fibrosis: Correlations with cardiopulmonary exercise test, 6-minute walk test, and quadriceps strength. Respiratory Care, Dallas, v. 61, n. 12, p 1620-1628, 2016.

HODGSON, C. L. et al. Clinical review: early patient mobilization in the ICU. Critical Care, London, v. 17, n. 1, p. 207, 2013.

JEFFERY, M. M. et al. Trends in emergency department visits and hospital admissions in health care systems in 5 states in the first months of the COVID-19 pandemic in the US. JAMA Internal Medicine, Chicago, v. 180, n. 10, p. 1328-1333, 2020.

JIANG, W. et al. Mental stress induced myocardial ischemia and cardiac events. JAMA, [s.I.], v. 275, n. 21, p. 1651-1656, 1996

LANA, R. et al. Emergência do novo corona vírus(SARS-CoV-2) e o papel de uma vigilância nacional em saúde oportuna eefetiva. Cadernos de Saúde Pública, Rio de Janeiro, v. 36 n. 3, p. 1-5 2020.

LI, Q. et al. Early transmission dynamics in Wuhan, China, of novel coronavirus-infected pneumonia. New England Journal of Medicine, Boston, v. 382, n. 13, p. 1199-1207, 2020.

LOURES, D. et al. Estresse mental e sistema cardiovascular. Arquivos Brasileiros de Cardiologia, São Paulo, v. 78, n. 5, p. 525-530, 2002.

MAFHAN, M. M. et al. COVID-19 pandemic and admission rates for and management of acute coronary syndromes in England. Lancet Public Health, v. 396, n. 10248, p. 381-389, 2020. 
MANSUR, A. P.; FAVARATO, D. Mortality due to cardiovascular diseases in women and men in the five Brazilian regions, 1980-2012. Arquivos Brasileiros de Cardiologia, São Paulo, v. 107, n. 2, p. 137-146, 2016.

MELO, D. O. et al. COVID-19 e doença hipertensiva no Brasil: possibilidade de uma tempestade perfeita. Revista Brasileira de Epidemiologia, São Paulo, v. 23, e200062, 2020.

NEEDHAM, N. et al. Early physical medicine and rehabilitation for patients with acute respiratory failure: A quality improvement project. Archives of Physical Medicine and Rehabilitation, Chicaho, v. 91, n. 4, p. 536-542, 2010.

NORMANDO, P. G. et al. Redução na hospitalização e aumento na mortalidade por doenças cardiovasculares durante a pandemia da COVID-19 no Brasil. Arquivos Brasileiros de Cardiolologia, São Paulo, v. 116, n. 3, p. 371-380, 2021.

OSUCHOWSKI, M. F. et al. The COVID-19 puzzle: deciphering pathophysiology and phenotypes of a new disease entity. The Lancet Respiratory Medicine, [s.I.], v. 9, n. 6, p. 622-642, 2021.

PRICE, K. J. et al. A review of guidelines for cardiac rehabilitation exercise programmes: Is there an international consensus? European Journal of Preventive Cardiology, London, v. 23, n. 16, p. 1715-1733, 2016.

RANDAL, R. J. et al. Home-Based Cardiac Rehabilitation: A Scientific Statement From the American Association of Cardiovascular and
Pulmonary Rehabilitation, the American Heart Association, and the American College of Cardiology. Circulation, Dallas, v. 140, n. 1, p. E69-E89, 2019.

ROTH, G. A. et al. Global, regional and national age-sex-specific mortality for 282 causes of death in 195 countries and territories, 1980-2017: a systematic analysis for the Global Burden of Disease Study 2017. The Lancet, London, v. 392, n. 10159, p. 1736-1788, 2018.

SZWARCWALD, C. L. et al. ConVid pesquisa comportamental. Disponível em: https://convid.fiocruz.br/index.php?pag=principal. Acesso em: 5 ago. 2021.

THOMAS, P. et. al. Phisiotherapy management for COVID-19 in the acute hospital setting: clinical practice recommendations. Journal of Physiotherapy, Hawthorn, v. 66, n. 2, p. 73-82, 2020.

TONIOLO, M. et al. Unpredictable fall of severe emergent cardiovascular diseases hospital admissions during the COVID-19 pandemic: Experience of a single large center in northern Italy. Journal ofthe American Heart Association, [s.I.], v. 9, n. 13,e017122, 2020.

WANG, C. et al. Immediate psychological responses and associated factors during the initial stage of the 2019 coronavirus disease (COVID-19) epidemic among the general population in China. International Journal of Environmental Research and Public Health, Basel, v. 17, n. 5, p. 1729-1754, 2020.

Submetido em: 19/11/2021

Aceito em: 22/11/2021 